\title{
RNAi-Based Gene Silencing of RXLR Effectors Protects Plants Against the Oomycete Pathogen Phytophthora capsici
}

\author{
Wei Cheng, ${ }^{1,2, \dagger}$ Menglan Lin, ${ }^{2}$ Moli Chu, ${ }^{1}$ Guixiang Xiang, ${ }^{2}$ Jianwen Guo, ${ }^{2}$ Yan Jiang, ${ }^{2}$ Deyi Guan, ${ }^{2}$ \\ and Shuilin $\mathrm{He}^{2, \dagger}$ \\ ${ }^{1}$ Anhui Provincial Key Laboratory of the Conservation and Exploitation of Biological Resources/College of Life Sciences, \\ Anhui Normal University, Wuhu, Anhui 241000, China \\ ${ }^{2}$ National Education Minister Key Laboratory of Plant Genetic Improvement and Comprehensive Utilization/College of Agriculture, \\ Fujian Agriculture and Forestry University, Fuzhou, Fujian 350002, China
}

Accepted 17 February 2022.

Phytophthora capsici is a broad-host range oomycete pathogen that can cause severe phytophthora blight disease of pepper and hundreds of other plant species worldwide. Natural resistance against $P$. capsici is inadequate, and it is very difficult to control by most of existing chemical fungicides. Therefore, it is urgent to develop alternative strategies to control this pathogen. Recently, host-induced or spray-induced gene silencing of essential or virulent pathogen genes provided an effective strategy for disease controls. Here, we demonstrate that $\boldsymbol{P}$. capsici can effectively take up small interfering RNAs (siRNAs) from the environment. According to RNA-seq and quantitative reverse transcription PCR analysis, we identified four $P$. capsici RXLR effector genes that are significantly up-regulated during the infection stage. Transient overexpression and promote-infection assays indicated that RXLR1 and RXLR4 could promote pathogen infection. Using a virusinduced gene silencing system in pepper plants, we found that in planta-expressing RNA interference (RNAi) constructs that target $R X L R I$ or $R X L R 4$ could significantly reduce pathogen infection, while co-interfering $R X L R 1$ and $R X L R 4$ could confer a more enhanced resistance to $P$. capsici. We also found that exogenously applying siRNAs that target $R X L R 1$ or $R X L R 4$ could restrict growth of $P$. capsici on the pepper and Nicotiana benthamiana leaves; when targeting $R X L R 1$ and $R X L R 4$ simultaneously, the control effect was more remarkable. These data

${ }^{\dagger}$ Corresponding authors: W. Cheng; chengwei8503@163.com, and S. He; shlhe201304@aliyun.com

Wei Cheng and Menglan Lin contributed equally to this work.

Funding: This work was supported by grants from Anhui Provincial Key Laboratory of the Conservation and Exploitation of Biological Resources in Anhui Normal University (swzy202007), Scientific Research Foundation of Anhui Normal University (903/762131), Science and Technology Innovation Foundation of Fujian Agriculture and Forestry University (CXZX2020004A), and Excellent Youth Foundation of Fujian Agriculture and Forestry University (xjq201725).

*The $\boldsymbol{e}$-Xtra logo stands for "electronic extra" and indicates there are supplementary materials published online.

The author(s) declare no conflict of interest.

(c) (1) () $\odot$ Copyright $(9) 2022$ The Author(s). This is an open access article distributed under the CC BY-NC-ND 4.0 International license. suggested that RNAi-based gene silencing of RXLR effectors has great potential for application in crop improvement against $\boldsymbol{P}$. capsici and also provides an important basis for the development of RNA-based antioomycete agents.

Keywords: Capsicum annuum, effector, gene silencing, Nicotiana benthamiana, Phytophthora, RNAi

Oomycetes are eukaryotic microorganisms that morphologically resemble true fungi but are phylogenetically related to diatoms and brown algae of the kingdom Stramenopila (Tyler et al. 2006). The Phytophthora genus is made up of plant-damaging oomycetes, which are notorious destroyers that cause enormous crop losses and environmental damage worldwide (Kamoun et al. 2015). Phytophthora blight of pepper, which caused by Phytophthora capsici, frequently reaches epidemic levels and causes huge yield losses of greater than $80 \%$ during severe local epidemics (Lamour et al. 2012a). P. capsici can infect all parts of the pepper plant, including roots, stems, leaves, and fruits at all growth stages, which causes seedling damping off, root rot, crown rot, stem blight, leaf spot, and fruit rot. For pepper seedlings, damping off associated with root rot can kill pepper plants 2 to 5 days after inoculation (Barchenger et al. 2018). Moreover, $P$. capsici is a broad-host range pathogen that can attack hundreds of plant species, including many other solanaceous plants, legumes, and most cucurbits (Lamour et al. 2012a).

Pepper (Capsicum annuum) is an important vegetable and spice crop worldwide. To date, natural resistance against $P$. capsici in pepper is inadequate. The pepper landrace Criollo de Morelos 334 (CM334) is the best-known source of resistance to $P$. capsici in the world. Although most of the commercial cultivars released nowadays have a certain degree of resistance to $P$. capsici, a higher level of resistance has not been observed (Barchenger et al. 2018; Kamoun et al. 2015; Mo et al. 2014). Studies on the genetics of resistance to $P$. capsici have led to the identification of several major quantitative trait loci (Barchenger et al. 2018). Considerable efforts have been made in the past few decades; however, a resistance gene against $P$. capsici in pepper plants has not been identified. Additionally, although morphologically similar to fungi, oomycetes are genetically and biochemically divergent (Tyler et al. 2006), thus, are generally not sensitive to most broad-spectrum fungicides (Barchenger et al. 2018). The fungicides providing systemic protection against oomycete diseases, including those caused by Phytophthora spp., are limited. Some 
chemical compounds, such as mefenoxam and metalaxyl, have been widely used to manage $P$. capsici. However, insensitivity is often observed soon after the first use of such compounds to manage the disease (Barchenger et al. 2018; Jackson et al. 2012). Therefore, the development of durable strategies to control this pathogen is a major challenge.

RNA interference (RNAi) is a conserved regulatory mechanism of gene expression in eukaryotic organisms and has been widely used to silence a gene of interest (Baulcombe 2004; Hannon 2002; Ketting 2011). Recently, increasing evidence has suggested that host-induced or spray-induced gene silencing (HIGS or SIGS) of the essential or virulent fungal pathogen genes could provide an effective and eco-friendly strategy for disease controls (Cai et al. 2018; Koch and Kogel 2014; Wang and Dean 2020; Wang and Jin 2017). HIGS refers to in planta-expressed RNAi constructs that target pathogen transcripts (Nowara et al. 2010; Nunes and Dean 2012), and SIGS refers to spraying double-stranded or small interfering RNA (dsRNA or siRNA) molecules on plant surfaces that target pathogen genes to reduce the severity of diseases (Koch et al. 2016; Wang et al. 2016). In plant-Phytophthora pathosystems, as a proof of concept, Jahan et al. (2015) generated transgenic potato plants expressing a hairpin RNA construct that target the green fluorescent protein (GFP) marker gene and detected a reduction of signal intensity of a corresponding GFP-expressing $P$. infestans strain inoculated on leaf samples of the transgenic plants. Furthermore, it was indicated that HIGS of a $G$ protein $\beta$-subunit (PiGPB1) gene or a RXLR effector (Avr3a) gene from $P$. infestans could confer potato resistance to late blight disease (Jahan et al. 2015; Sanju et al. 2015), which demonstrated that the HIGS method can confer disease control against a Phytophthora pathogen.

During pathogenesis, phytopathogens usually secrete effectors into the plant apoplast or intracellular compartments to modulate host immunity to favor successful infection. Recently, an increasing number of studies have reported that Phytophthora effectors, particularly RXLR effectors, named after an N-terminal RXLR amino acid motif, are able to manipulate diverse host immune processes (He et al. 2020; Jiang and Tyler 2012). For example, different $P$. capsici RXLR effectors could target a variety of host proteins, such as FKBP15-2 (an endoplasmic reticulum-localized PPIase), ACD11-binding partners, NPR1 (nonexpressor of pathogenesis related-1), EDS1 (enhanced disease susceptibility 1), and CAD7 (cinnamyl alcohol dehydrogenase 7) subfamily members, to suppress plant immunity in order to promote disease (Fan et al. 2018; Li et al. 2019a, b, and c, 2020).

As Phytophthora RXLR effectors are critical modulators in pathogenicity, the main objective of this study was to investigate the possibility of exploiting RNAi-based gene silencing of RXLR effectors to protect plants against $P$. capsici. First, we determined whether $P$. capsici could take up dsRNA or siRNAs from the environment. Then, $P$. capsici RXLR effector genes that are significantly induced at the infection stage were identified by RNA-seq and quantitative reverse transcription PCR (qRT-PCR) analysis and tested to promote the pathogen infection. Subsequently, we selected two of these RXLR effector genes (RXLRI and $R X L R 4$ ) for RNAi targets and investigated whether in-planta expression of the RNAi constructs or exogenously applying siRNAs that target one or both RXLRI and RXLR4 could restrict growth of $P$. capsici.

\section{RESULTS}

$P$. capsici could effectively take up environmental siRNAs.

To investigate whether $P$. capsici can take up siRNAs from the environment, $5^{\prime}$ carboxyfluorescein (FAM)-labeled siRNA duplexes (24 nt) were applied on $P$. capsici germinated cysts and mycelia and were grown on liquid 1/100 V8 medium. The fluorescent signals from germinated cysts and mycelia were detected after treatment at $0.5,1,2,4,6$, and $12 \mathrm{~h}$ intermittently. Strikingly, fluorescent signals were rapidly accumulated into $P$. capsici cells for both germinated cysts and mycelia within $0.5 \mathrm{~h}$ postincubation and reached their maximum concentrations at $4 \mathrm{~h}$ postincubation (Fig. 1). To exclude the possibility that $5^{\prime}$ FAM siRNAs adhered to an exterior site on $P$. capsici cells, the germinated cysts and mycelia were also treated with micrococcal nuclease (MNase) (Wang et al. 2016) and PBT solution ( $1 \times$ phosphate saline buffer containing $0.3 \%$ Triton X-100), respectively, and the fluorescent signals remained detectable without reduction. Additionally, we also synthesized 5' FAM-labeled long dsRNA duplexes (151 nt) and applied them on P. capsici germinated cysts and mycelia. Consistent with a recent report on $P$. infestans (Qiao et al. 2021), no or very weak fluorescence signals were observed in $P$. capsici germinated cysts and mycelia treated with the fluorescein-labeled dsRNAs even after $12 \mathrm{~h}$ postincubation (data not shown). These results indicated that $P$. capsici could effectively take up siRNAs rather than long dsRNAs from the environment.

\section{Identification of $P$. capsici RXLR effectors that contribute to promoting colonization.}

Based on RNA-seq and qRT-PCR analysis, we identified four $P$. capsici RXLR effector genes (named RXLRI to RXLR4) that are significantly up-regulated during the stages of infection, whereas they exhibit relative low transcript levels at the mycelium stage cultured in vitro (Fig. 2; Supplementary Fig. S1). We then cloned these four $P$. capsici RXLR effector genes without signal peptide sequences into plant overexpression vector pBinGFP2, respectively. Transient overexpression of these RXLR effectors could not induce cell death response both in pepper and in Nicotiana benthamiana leaves (Supplementary Fig. S2). Then, these RXLR effectors were used for identification of their contributions to promoting colonization. Transient overexpression of these RXLR effectors in pepper or $N$. benthamiana leaves and subsequent inoculation with $P$. capsici indicated that $R X L R I$ and $R X L R 4$ could significantly promote pathogen growth, whereas $R X L R 2$ and RXLR3 could not (Fig. 3; Supplementary Fig. S3). Thus, we selected $R X L R I$ and $R X L R 4$ for RNAi targets in the subsequent study.

\section{HIGS of $R X L R 1 / R X L R 4$ could reduce phytophthora blight disease.}

Pepper is very recalcitrant to generate stable transgenic plants by genetic transformation approaches. To determine whether HIGS of one or both RXLR1 and RXLR4 could control phytophthora blight of pepper, we introduced the specific RXLR1/RXLR4RNAi fragments into pepper plants by a virus-induced gene silencing (VIGS) system (Fig. 4A). The TRV:0 (empty vector) treated pepper plants served as negative controls. And the TRV:CaPDStreated pepper plants, which silenced the phytoene desaturase (PDS) gene, were used as indicator controls. Three weeks after agroinfiltration, the TRV:CaPDS-treated plants showed the silencing (photobleaching) phenotype (Supplementary Fig. S4). Then, the tobacco rattle virus (TRV)-treated pepper plants were inoculated with $P$. capsici for disease assessment.

For detached leaf assays, the leaves from each TRV-treated pepper plant were inoculated with $P$. capsici mycelial plugs. The transcriptional expression of $R X L R I$ and $R X L R 4$ was reduced by 62 to $74 \%$ in $P$. capsici grown on the corresponding VIGS pepper leaves (Fig. 4B). After $P$. capsici inoculation, the detached VIGS pepper leaves targeting either $R X L R l$ (TRV:RXLRl) or RXLR4 (TRV:RXLR4) exhibited decreased disease symptoms as compared with the TRV: 0 controls (Fig. 4C). The relative $P$. capsici biomass in the TRV:RXLRI- and TRV:RXLR4-treated leaves was decreased, respectively, by approximately 81 and $57 \%$, as compared with that in the TRV:0 controls (Fig. 4D). Furthermore, the detached VIGS pepper leaves targeting RXLR1 and RXLR4 
(TRV:RXLR1\&4) displayed much smaller lesions and less $P$. capsici growth. The pathogen biomass in the TRV:RXLRI\&4 treated leaves was decreased by approximately $85 \%$, as compared with that in the TRV: 0 controls (Fig. $4 \mathrm{C}$ and D). We also rootinoculated the intact VIGS pepper plants with $P$. capsici zoospores and found that TRV:RXLRI- and TRV:RXLR4-treated plants, and particularly TRV:RXLRI\&4-treated plants, exhibited significantly alleviated disease symptoms as compared with the TRV:0 controls (Fig. 4E and F). These results indicated that in planta-expressing the RNAi constructs that target $R X L R I$ and $R X L R 4$ could efficiently silence the transcripts of the corresponding RXLR effector genes, which led to suppression of $P$. capsici virulence and inhibition of the disease.

\section{SIGS of $R X L R I / R X L R 4$ could restrict growth of $P$. capsici.}

Encouraged by the results above, we next explored whether one or more environmental RXLR effectors targeting siRNAs could restrict growth of $P$. capsici. One or both the synthetic $R X L R I$-siRNAs and RXLR4-siRNAs or control-siRNAs (40 ng/ $\mu \mathrm{l}$ ) were applied onto the detached leaves of pepper and $N$. benthamiana plants, followed by $P$. capsici inoculation with mycelial plugs or zoospores. The transcriptional expression of $R X L R 1$ and $R X L R 4$ was successfully silenced in $P$. capsici collected from the corresponding leaves treated with $R X L R 1$-siRNAs, $R X L R 4$-siRNAs, or both, as compared with those from leaves treated with GFP control-siRNAs (Fig. 5A and D). The pepper and $N$. benthamiana leaves treated with $R X L R 1$-siRNAs or $R X L R 4$-siRNAs exhibited smaller lesions and less $P$. capsici biomass, whereas the controls showed severe disease lesions and relative higher pathogen biomass. When treated with RXLRI-siRNAs and RXLR4-siRNAs simultaneously at the same amount, both the pepper and $N$. benthamiana leaves displayed very mild disease symptoms, exhibiting much smaller lesions and less $P$. capsici growth (Fig. 5B and C). These results indicated that exogenously applying siRNAs that target RXLRI or RXLR4 could restrict growth of $P$. capsici on the plant surface; when targeting RXLRl and RXLR4 simultaneously, the control effect was more remarkable.

\section{DISCUSSION}

The development of enhanced $P$. capsici resistance traits in pepper and other vegetable crops has been a great challenge, due to the limited availability of resistant germplasms and the rapid breakdown of natural resistance by the pathogen with genetic variations (Barchenger et al. 2018; Kamoun et al. 2015). Furthermore, the efficacy of cultural practices and chemical controls for management of phytophthora blight is limited (Barchenger et al. 2018; Jackson et al. 2012; Lamour et al. 2012a; Mo et al. 2014). Therefore, there is an urgent demand to

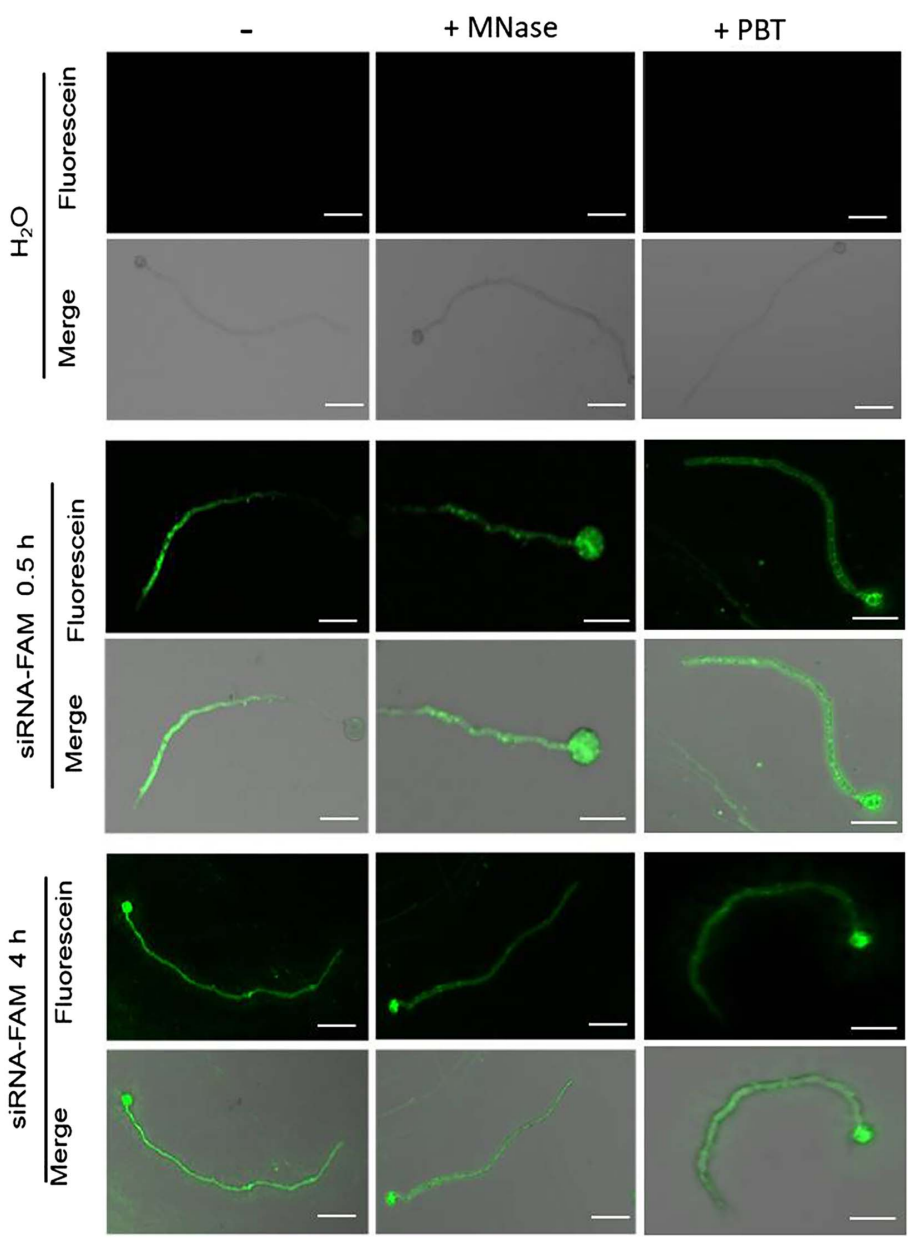

GC

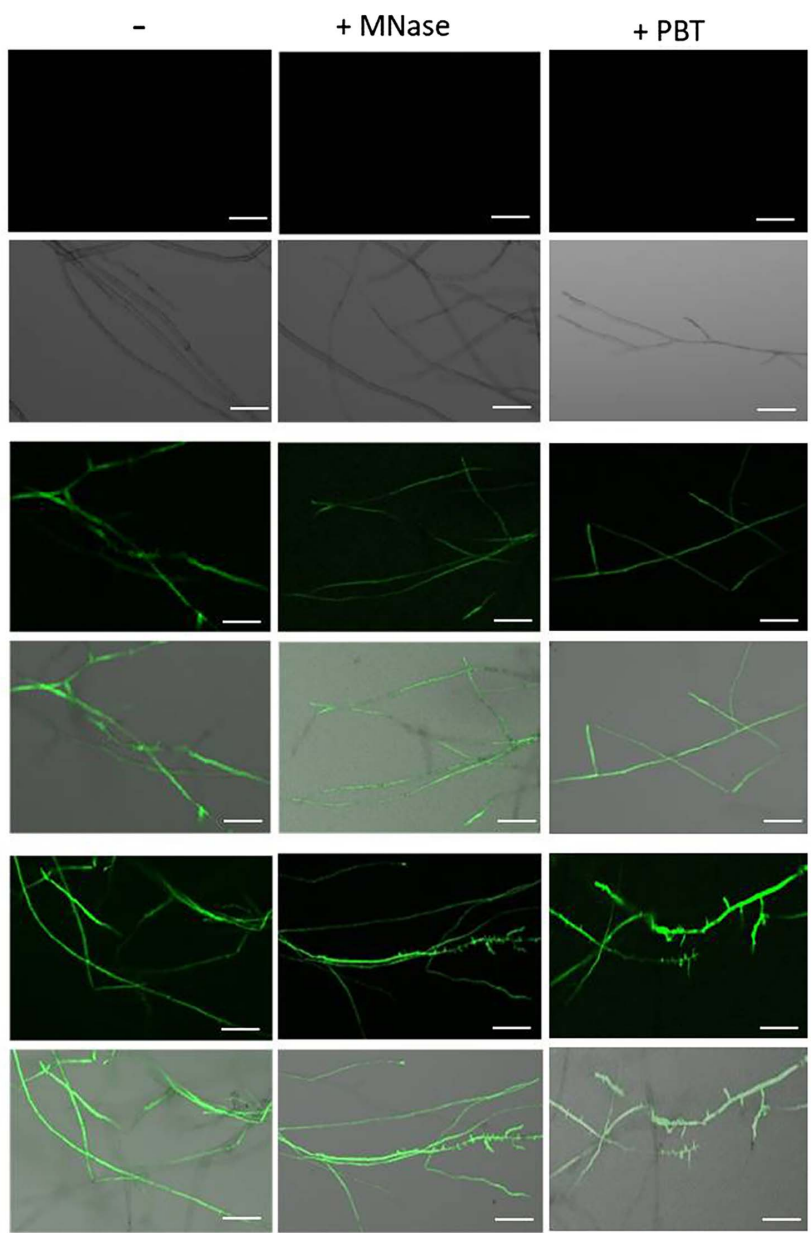

MY

Fig. 1. Phytophthora capsici could effectively take up environmental small interfering (si)RNAs. To monitor whether $P$. capsici can take up siRNAs from their environment, $5^{\prime}$ FAM-siRNAs (24 nt) were applied on the germinated cysts (GC) and mycelia (MY) of $P$. capsici, which grown on liquid 1/100 V8 medium. The fluorescent signals of GC and MY were observed using fluorescence microscopy after treatment with or without micrococcal nuclease (MNase) and 1× phosphate saline buffer containing 0.3\% Triton X-100 (PBT) solution. Each experiment was repeated three times with similar results. Scale bar $=25 \mu \mathrm{m}$. 
develop alternative strategies to control this pathogen. Encouraged by the proof of concept for HIGS of target genes from $P$. infestans (Jahan et al. 2015; Sanju et al. 2015) and our results showing that $P$. capsici could effectively take up siRNAs from the environment (Fig. 1), we investigated the possibility of exploiting HIGS and SIGS methods to protect plants against $P$. capsici. For $P$. infestans, Qiao et al. (2021) indicated that after incubating with fluorescein-labeled dsRNA, weak fluorescent signals were detected in some $P$. infestans hyphae and no signal was observed in hyphae derived from the germination of zoospores $12 \mathrm{~h}$ postinoculation. Similarly, in our experiments, no or very weak fluorescent signals were observed in $P$. capsici germinated cyst and hyphae treated with 5' FAM-labeled dsRNAs, even $12 \mathrm{~h}$ postincubation. These results indicated that dsRNA uptake was limited in Phytophthora spp. whereas, after incubating 5' FAMlabeled siRNAs, the fluorescent signals were rapidly accumulated into $P$. capsici cells for both germinated cysts and mycelia within $0.5 \mathrm{~h}$ postincubation and reached the maximum concentrations at $4 \mathrm{~h}$ postincubation. These data suggested that Phytophthora spp. could effectively take up siRNAs rather than longer dsRNAs from the environment. The high efficiency of environmental siRNA uptake may be due to its characteristics of low molecular weight and ready delivery. However, whether the transport of siRNAs or dsRNAs through cell membranes is driven by specific delivery vehicles or accessory factors is, so far, unknown.

The RXLR effector genes were selected for RNAi targets because increasing evidence suggests that Phytophthora RXLR effectors are critical modulators for pathogen pathogenicity (He et al. 2020; Jiang and Tyler 2012). Thus, four $P$. capsici RXLR effector genes that are significantly induced during the infection stage were identified by RNA-seq and qRT-PCR analysis. Screened by transient overexpression and promoting infection assays, $R X L R I$ and $R X L R 4$ were verified to promote pathogen infection, indicating that they are able to suppress plant immunity to promote disease. Further work is necessary to dissect their host targets and the associated modes of action of these RXLR effectors, particularly for RXLR1 and RXLR4.

To determine whether HIGS of RXLR effectors could reduce the severity of phytophthora blight disease, we employed the TRV-based VIGS system and found that in planta-expressing the RNAi construct that targets RXLRI or RXLR4 could effectively reduce pathogen infection, while silencing of $R X L R 1$ and $R X L R 4$ simultaneously could confer the pepper plants a more enhanced resistance to $P$. capsici. Previous study indicated that HIGS of a $P$. infestans RXLR effector (Avr $3 a$ ) gene could confer potato resistance to the late blight disease (Sanju et al. 2015). Moreover, Vega-Arreguín et al. (2014) revealed that knockdown of the expression of a $P$. capsici RXLR effector (PcAvr3al) by HIGS allowed the infection of $P$. capsici in the nonhost tobacco (Nicotiana tabacum) plants. In line with these previous studies, we suggest that RXLR effectors can be selected as RNAi targets to protect plant against the Phytophthora pathogen.

To circumvent transgenic approaches, direct spray of siRNAs that target $R X L R 1$ and $R X L R 4$ onto the pepper and $N$. benthamiana leaves led to silencing of one or more target genes and conferred effective disease control of $P$. capsici. Together with the HIGS method, we found that SIGS of RXLR1 and RXLR4 simultaneously showed a more enhanced resistance against $P$. capsici, indicating that HIGS or SIGS of multitarget virulent genes may lead to an additive or synergistic effect of disease controls. It is worth noting that with the availability of the $P$. capsici genome, over 350 putative RXLR effector candidates have been identified (Lamour et al. 2012b; Reyes-Tena et al. 2019). HIGS or SIGS can be used as a tool to screen potentially crucial effectors or other pathogenicity genes to understand the interactions between host and the pathogen.
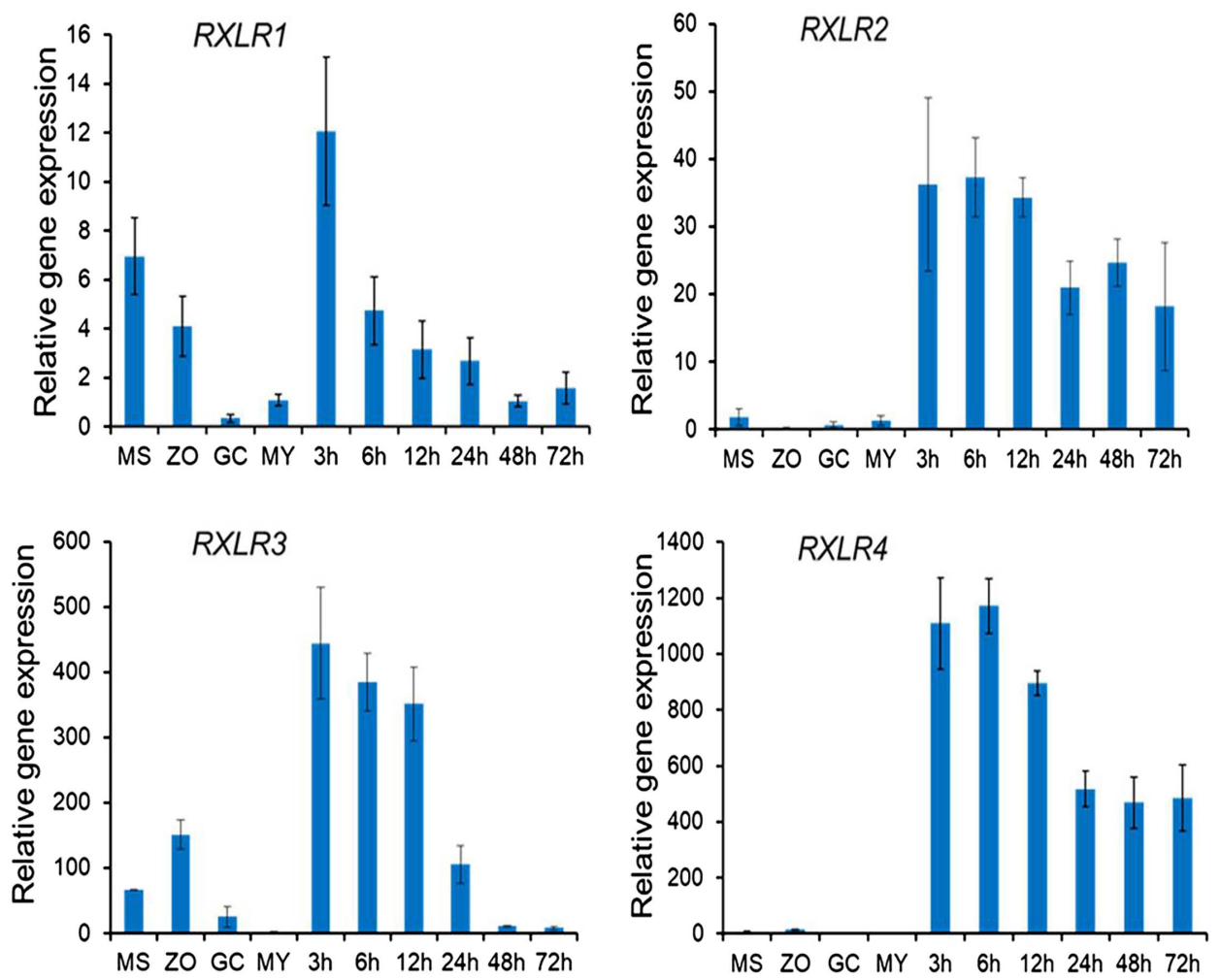

Fig. 2. The transcriptional profiles of Phytophthora capsici RXLR effectors. Transcript levels of four P. capsici RXLR effector genes (RXLR1 to RXLR4) were measured by quantitative reverse transcription PCR analysis. Samples were collected from various life cycle stages, including mycelium (MY), sporangium (MS), zoospore (ZO), and germinated cyst (GC) cultured in vitro, and at the indicated timepoints during the infection stage. Means and standard errors were calculated from three independent biological replicates. 


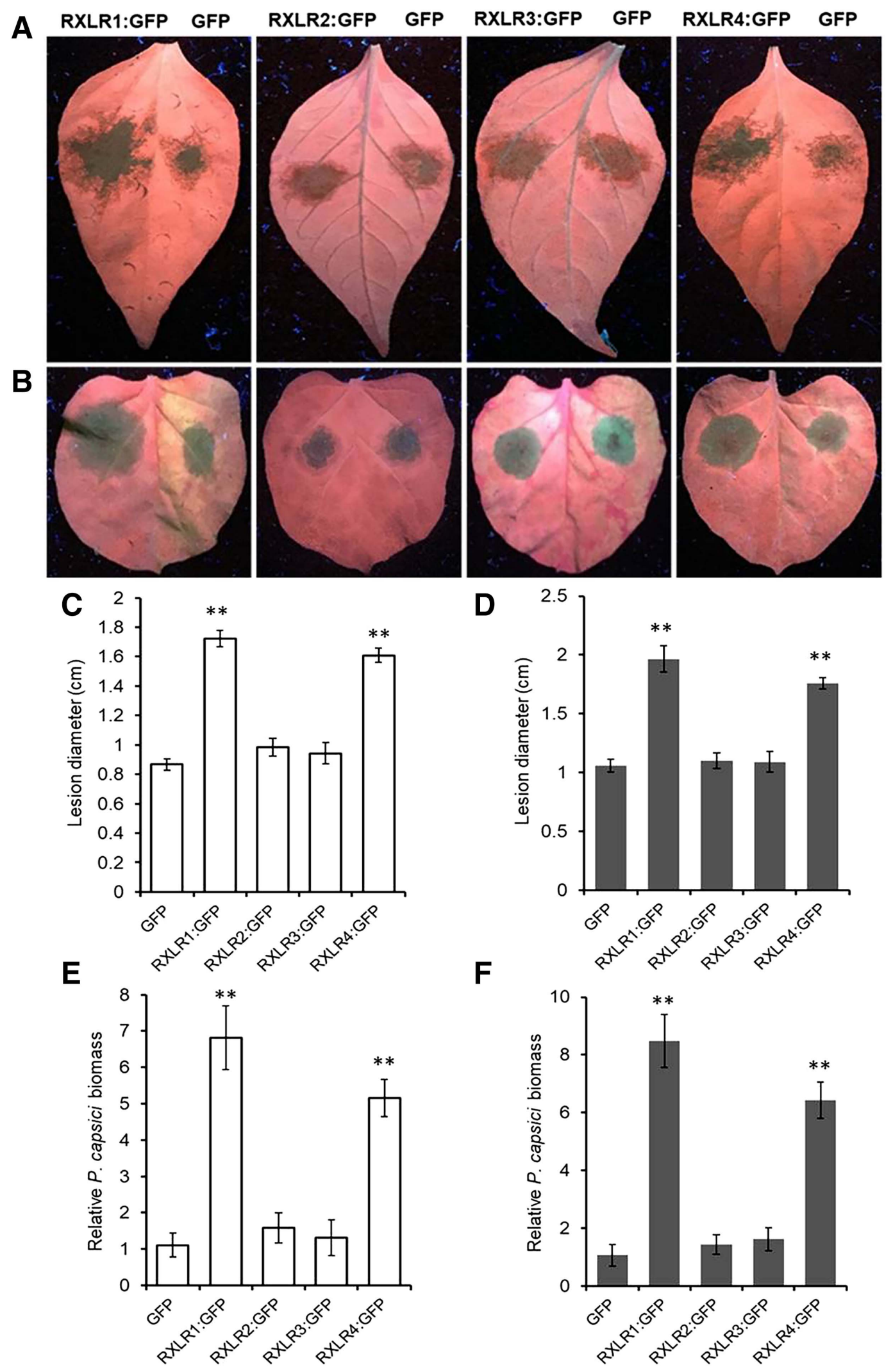

Fig. 3. Identification of Phytophthora capsici RXLR effectors that contribute to promoting colonization. For transient overexpression and promote-infection assays, the leaves of pepper and Nicotiana benthamiana were infiltrated with Agrobacterium tumefaciens GV3101 harboring pBinGFP2-RXLR1, pBinGFP2-RXLR2, pBinGFP2-RXLR3, and pBinGFP2-RXLR4, respectively. The empty vector (pBinGFP2) served as negative control. One day after agroinfiltration, these leaves were subsequently inoculated with $P$. capsici mycelial plugs and were kept at high humidity in the dark at $25^{\circ} \mathrm{C}$ for 2 days prior to assessment. A and B, Disease symptoms on pepper and $N$. benthamiana leaves, respectively, were photographed under UV light at 2 days postinoculation (dpi). C to F, Lesion diameters and relative $P$. capsici biomass were measured in the leaves of pepper (C and E) and $N$. benthamiana (D and F) to evaluate the severity of infection at $2 \mathrm{dpi}$. Asterisks indicate statistically significant differences compared with the controls by the least significant difference test (two asterisks $\left.{ }^{* *}\right]$ indicate $P<0.01$ ). Each experiment was repeated twice, each time with five independent biological replicates. 
Current disease control measures mainly rely on the application of chemical fungicides, which potentially produce resistant pathogen strains as well as other undesirable environmental and health side-effects. RNAi-based gene silencing (HIGS or SIGS) disease controls seem to be more sustainable and do not easily induce resistant mutated pathogen strains to escape RNA silencing, because effective RNAi is sequence-based rather than structurebased and does not require perfect base pairing (Wang and Jin 2017; Waterhouse et al. 2001). Moreover, due to their sequencespecific dependent manner, HIGS or SIGS can be tailored to be more effective when targeting multiple pathogen genes and can also potentially be developed against an unlimited range of pathogens by targeting multiple genes that are essential or virulent or both from different pathogens (Wang and Jin 2017). Although RNA-based agents in SIGS remain effective for up to about one week after treatment (Koch et al. 2016; Wang et al. 2016), there is demand for longer lasting agronomic applications. In mammalian systems, several studies have indicated that siRNA modifications, such as 2'-methoxy, 2'-fluoro, 2'-O-benzyl, or 2'-O-methyl-4-pyridine, could increase the duration and potency of siRNAs in vivo compared with their unmodified counterparts (Kenski et al. 2012). So, it is possible that siRNA-optimized modifications, by mixing with stabilizing agents or surfactants, could improve their half-lives and effective periods (Dubrovina and Kiselev 2019).

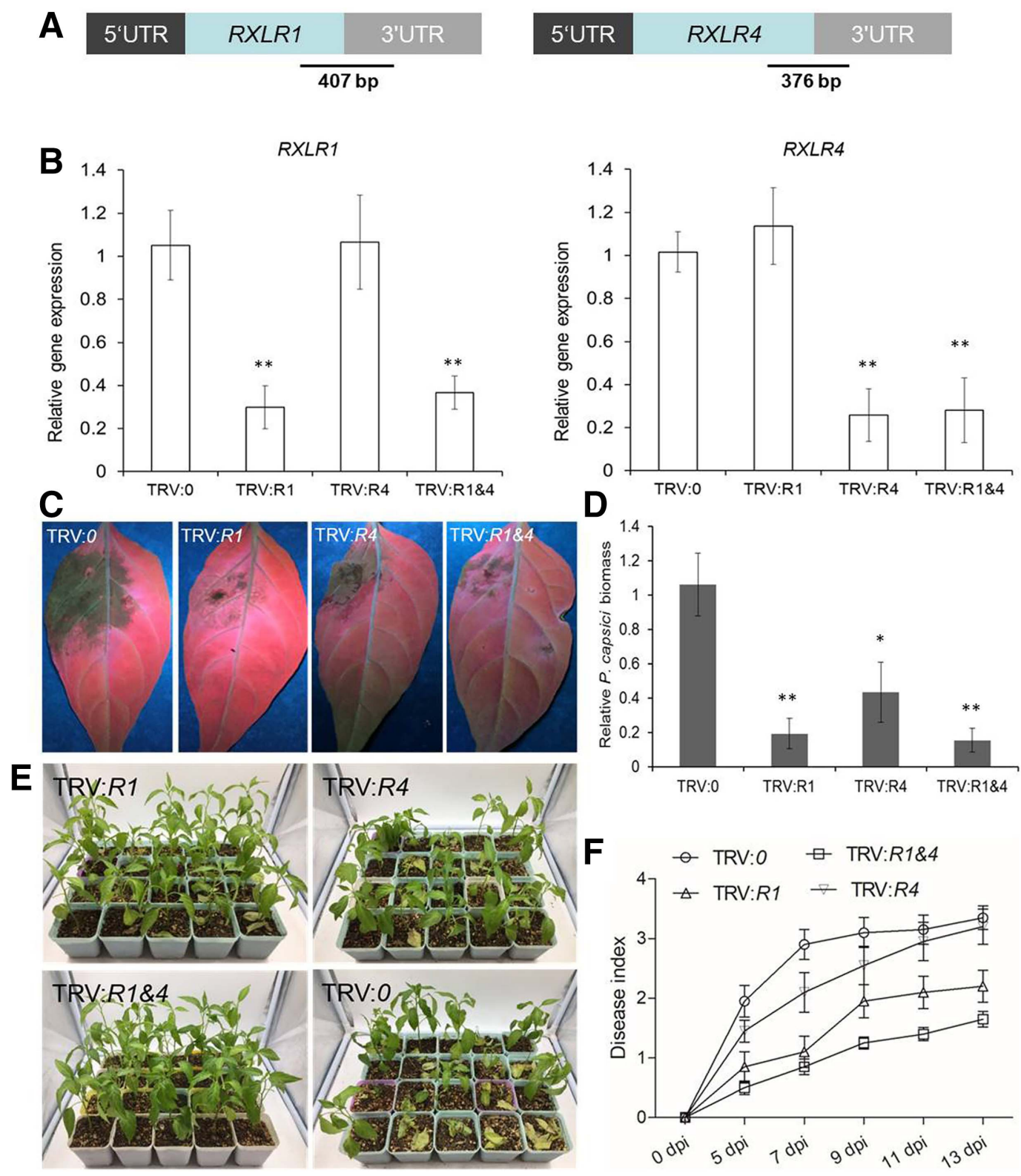

Fig. 4. Host-induced gene silencing of RXLR1/RXLR4 protects pepper plants against Phytophthora capsici. A, To determine whether in plantaexpressing the RNA interference (RNAi) constructs that target $R X L R 1 / R X L R 4$ could protect pepper plants against $P$. capsici, one or both of the specific RXLR1 or RXLR4-RNAi fragments were introduced into the pepper plants by tobacco rattle virus (TRV)-based virus-induced gene silencing system. The TRV:O (empty vector)-treated pepper plants served as negative controls. For detached leaf assays, the leaves from each TRV-treated pepper plant were inoculated with $P$. capsici mycelial plugs. B, The silencing efficiency of RXLRI and RXLR4 was determined by quantitative reverse transcription PCR analysis at 1 day postinoculation (dpi). C, Disease symptoms were photographed under UV light and $\mathbf{D}$, relative $P$. capsici biomass was measured at 2 dpi. Asterisks indicate statistically significant differences compared with the TRV:0 empty vector controls by the least significant difference test (one asterisk $\left[{ }^{*}\right]$ indicates $P<0.05$, two $\left.{ }^{* *}\right] P<0.01$ ). E, The phenotypes of these pepper plants were photographed at 7 days after the root-inoculation assay, for which the growing pepper plants were inoculated with $5 \mathrm{ml}$ of $P$. capsici zoospores $\left(5 \times 10^{5}\right.$ per milliliter). F, The disease indexes of these pepper plants were assessed after roots were inoculated at 5, 7, 9, 11, and 13 dpi. 


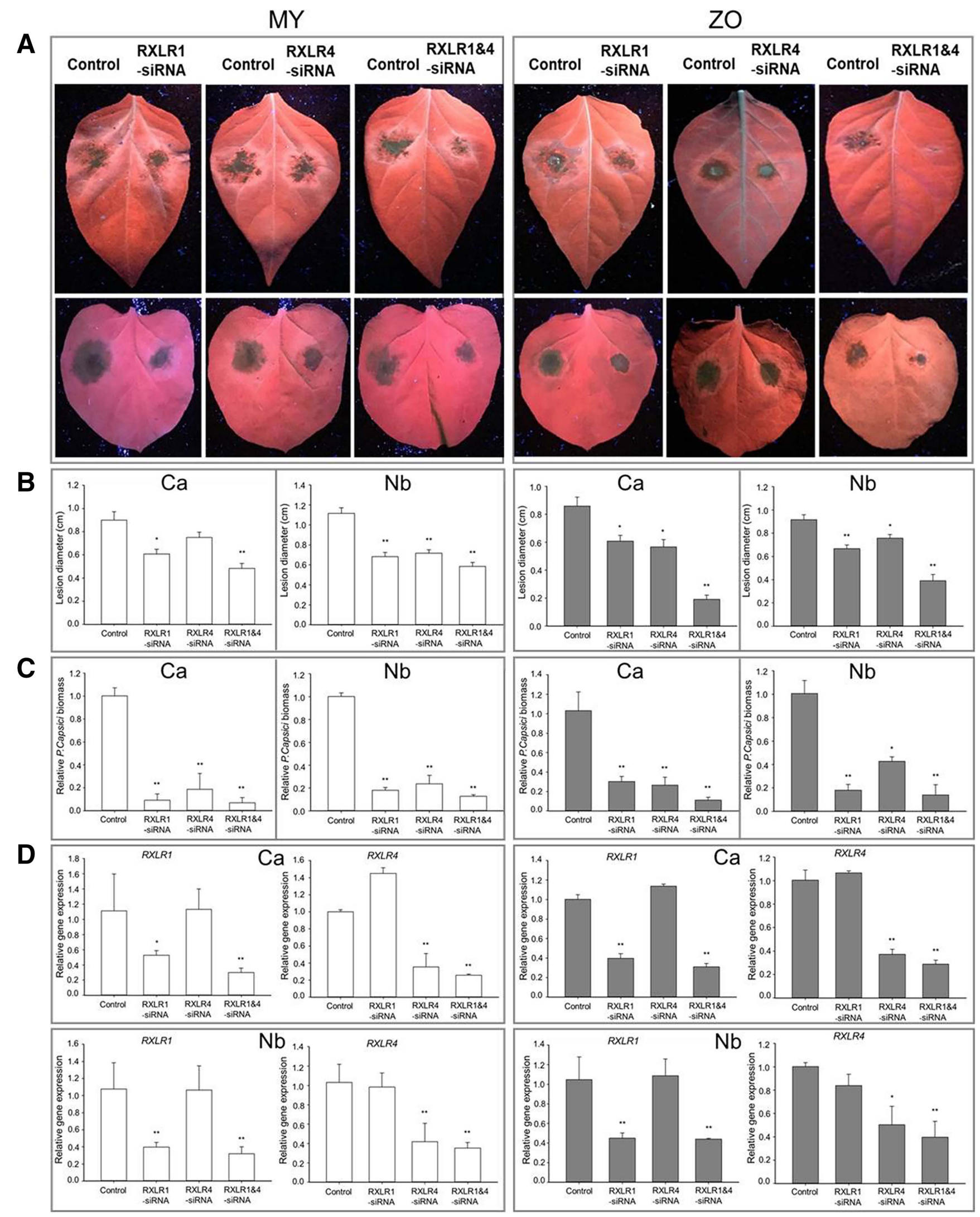

Fig. 5. Spray-induced gene silencing of RXLR1/RXLR4 could restrict growth of Phytophthora capsici. To determine whether exogenously applied small interfering (si)RNAs that target $R X L R 1 / R X L R 4$ could restrict growth of $P$. capsici on the plant surface, $20 \mu 1$ of synthetic $R X L R 1$-siRNAs, RXLR4-siRNAs, or both or control-siRNAs $(40 \mathrm{ng} / \mu \mathrm{l})$ were dropped onto the detached pepper or $N$. benthamiana leaves, followed by $P$. capsici inoculation with mycelial plugs or $3 \mu \mathrm{l}$ of zoospores $\left(5 \times 10^{5}\right.$ per milliliter). A, Disease symptoms of $N$. benthamiana and pepper leaves were photographed under UV light after inoculation with mycelial plugs (MY), at 2 days postinoculation (dpi), and zoospores (ZO), at 3 dpi. B and $\mathbf{C}$, Lesion diameters and relative P. capsici biomass of the N. benthamiana and pepper leaves, respectively, were measured. D, The transcriptional expression of $R X L R 1$ and $R X L R 4$ was determined by quantitative reverse transcription PCR analysis at 1 day postinoculation. Asterisks indicate statistically significant differences, compared with the controls, by the least significant difference test (one asterisk [*] indicates $P<0.05$, two [**] $P<0.01$ ). Each experiment was repeated twice, each time with five independent biological replicates. 
Recently, it is strikingly reported that several nanoparticles have the potential to increase the efficacy and duration of plant protection by environmental RNAi (Mitter et al. 2017; Wang et al. 2020). In addition, in-vitro RNA synthesis is currently too costly for large-scale production. Therefore, further investigations are necessary to solve these issues for its practical application under field conditions.

In conclusion, the data presented in this study suggest that RNAi-based gene silencing of Phytophthora-specific virulence genes has great potential for application in crop improvement against phytophthora blight and also provides an important basis for the development of RNA-based antioomycete agents.

\section{MATERIALS AND METHODS}

\section{Strains, plants, and culture conditions.}

$P$. capsici JX1 was isolated by our laboratory and cultured on $10 \% \mathrm{~V} 8$ agar medium at $25^{\circ} \mathrm{C}$ in the dark. To induce sporangial production and zoospore release, mycelia of $P$. capsici were cultured on $10 \%$ liquid V8 medium for approximately 3 days, were then washed with sterilized water three times intermittently, and were incubated at $25^{\circ} \mathrm{C}$ until zoospores were produced (Cheng et al. 2019). The seeds of pepper cultivar 130 and N. benthamiana were sown in a soil mix (peat moss and perlite, 2:1 [vol/ vol]) in plastic pots and were placed in a growth room under the conditions of $25^{\circ} \mathrm{C}$, relative humidity of $70 \%$, 60 to $70 \mathrm{mmol}$ photons per square meter per second, and a 16-h light and 8-h dark photoperiod (Cheng et al. 2017).

\section{Fluorescein siRNA, migration assays, and microscopy analysis. \\ $5^{\prime}$ FAM-labeled 24-nt siRNA duplexes (5'-CACAAGUUCA} GCGUGUCCGGCGAG-3') and 151-nt dsRNA duplexes, both derived from GFP coding sequence, were synthesized by the GenePharma Company (Shanghai, China). To monitor whether $P$. capsici can take up siRNAs or dsRNAs from the environment, 5' FAM-labeled siRNAs or dsRNAs (final concentration of $40 \mathrm{ng} / \mu \mathrm{l}$ ) were applied on the germinated cysts and mycelia of $P$. capsici, respectively, and were grown on liquid $1 / 100 \mathrm{~V} 8$ medium. After intermittent treatment for $0.5,1,2,4,6$, and $12 \mathrm{~h}$, the fluorescent signals of germinated cysts and mycelia were observed, using a laser scanning confocal microscope (TCS SP8, Leica, Solms, Germany). To exclude the possibility that the fluorescent signals adhered to the exterior site of the $P$. capsici cells, the germinated cysts and mycelia described above were incubated with 75 U MNase (Thermo Fisher Scientific, Waltham, MA, U.S.A.) at $37^{\circ} \mathrm{C}$ for $30 \mathrm{~min}$ (Wang et al. 2016) or were washed three times with PBT solution before confocal microscopy.

\section{RNA-seq and qRT-PCR analysis.}

$P$. capsici RNA-seq data were obtained from various stages of the life cycle including the mycelium, sporangium, zoospore, and germinated cyst as well as pepper roots at 3, 6, 12, 24, 48, and $72 \mathrm{~h}$ after inoculation with JX1 zoospores (Cheng et al. 2020). The RNA-seq data are available in the National Center for Biotechnology Information Sequence Read Archive under accession number PRJNA627546. For qRT-PCR analysis, total RNA was extracted and first-strand cDNA was synthesized as described previously (Cheng et al. 2019). To determine the relative transcription levels of each RXLR effector gene, realtime PCR was performed with the specific primers listed in Supplementary Table S1. Three independent biological replicates of each treatment were performed. The data were analyzed using the Livak method and were calculated as a normalized relative expression level $\left(2^{-\Delta \Delta C T}\right)$ (Livak and Schmittgen 2001).
Transient overexpression and promoting-infection assays.

P. capsici RXLR effector genes RXLR1 (PHYCA_20942), RXLR2 (PHYCA_114125), RXLR3 (PHYCA_12939), and RXLR4 (PHYCA_107349), which are significantly up-regulated during the infection stage as compared by RNA-seq analysis with the mycelium cultured in vitro (at least one infection timepoint with fragments per kilobase per million reads $>10$, fold change $>2$, and false discorvery rate $\leq 0.01$ ), were amplified from cDNA of $P$. capsici-infected pepper tissues with the primers listed in Supplementary Table S1. The purified fragments without signal peptide sequences were then cloned into pBinGFP2 plasmids using an In-Fusion HD cloning kit (Clontech, Mountain View, CA, U.S.A.) and were sequenced.

For transient overexpression analysis, Agrobacterium tumefaciens GV3101 harboring the pBinGFP2-RXLR1, pBinGFP2RXLR2, pBinGFP2-RXLR3, pBinGFP2-RXLR4, or pBinGFP2 (empty vector used as a control) was resuspended in the induction medium (optical density at $600 \mathrm{~nm}\left[\mathrm{OD}_{600}\right]=0.8$ ) and was then infiltrated into the leaves of pepper or $N$. benthamiana plants at the eight-leaf stage, using a syringe without needle. At 3 to 5 days post agroinfiltration, these leaves with point injection were observed for cell-death response. For the promoting-infection assays, after 1 day post agroinfiltration, these leaves were subsequently inoculated with $P$. capsici for infection assays. Each experiment was repeated twice, each time with five independent biological replicates.

\section{The VIGS vectors construction.}

To construct a VIGS vector of each selected RXLR gene, the specific silencing fragment was determined by BLAST analysis in the pepper and $P$. capsici genome cDNA database (Basenko et al. 2018; Fernandez-Pozo et al. 2015), and no off-target gene (one that shares no more than a 19-bp matching fragment) was detected. The specific 407- and 376-bp fragments of RXLRI and RXLR4, which were prepared from the junction regions of the open read frame and $3^{\prime}$ untranslated region, were amplified by PCR from cDNA of $P$. capsici-infected pepper tissues, using PrimeSTAR GXL DNA polymerase (Takara Bio Inc., Otsu, Japan) with the primers listed in Supplementary Table S1. The purified fragments were then cloned into the TRV silencing vector pTRV2 by Gateway technology (Life Technologies, Carlsbad, CA, U.S.A.).

\section{HIGS of RXLR effector genes \\ in pepper plants against $P$. capsici.}

For HIGS of RXLR effector genes in pepper plants, the TRVbased VIGS system was employed according to our previous studies (Cheng et al. 2017; Dang et al. 2013). Strains of A. tumefaciens GV3101 harboring pTRV1 and pTRV2: RXLR1 (or pTRV2: $R X L R 4)$ were resuspended in the induction medium $\left(\mathrm{OD}_{600}=0.8\right)$ and were mixed thoroughly with an equal volume and were then infiltrated into the two cotyledons of 2-week-old pepper plants. When targeting RXLR1 and RXLR4 in combination, A. tumefaciens strains harboring pTRV2: RXLR1 and pTRV2: RXLR4 were first mixed at a 1:1 (vol/vol) ratio, then mixed with pTRV1 in an equal volume. pTRV2:0 (empty vector) served as a negative control. pTRV2: $C a P D S$, which silences the PDS gene and induces a photobleaching phenotype, was used as an indicator control (Supplementary Fig. S4). The agroinfiltrated pepper plants were kept in an incubator in darkness at $16^{\circ} \mathrm{C}$ for $56 \mathrm{~h}$ and were then transferred into the growth room under normal conditions, as described above. One week later, the agroinfiltrated pepper plants described above were drenched with $6 \mathrm{ml}$ the same Agrobacterium culture mix and were grown for about three more weeks for further analysis (Ryu et al. 2004; Senthil-Kumar and Mysore 2014).

For VIGS pepper plants, the third and fourth detached leaves from the top of each TRV-treated pepper plant were inoculated with $P$. capsici mycelial plugs (Cheng et al. 2020). After $P$. capsici 
inoculation, the silencing efficiency of each RXLR gene was determined by qRT-PCR analysis using the fourth detached leaves at 1 day postinoculation (dpi), and the third detached leaves were kept at high humidity in the dark at $25^{\circ} \mathrm{C}$ for 2 to 3 days prior to disease assessment. We used qRT-PCR analysis to measure relative $P$. capsici biomass (the ratios of $P$. capsici DNA to host DNA in infected tissues). Infected plant tissues were harvested and total genomic DNA was extracted, using a DNeasy plant minikit (Qiagen, Hilden, Germany). The PcActin gene was used to target the pathogen, and the CaActin or $N t E F 1 \alpha$ was served as host target gene. The specific primer pairs were listed in Supplementary Table S1. Each experiment was repeated twice, each time with five independent biological replicates.

We also root-inoculated the growing pepper plants with $5 \mathrm{ml}$ of $P$. capsici zoospores $\left(5 \times 10^{5}\right.$ per milliliter $)$ and assessed the disease indexes of these pepper plants at $5,7,9,11$, and13 dpi. The disease index scores are 0 (asymptomatic), 1 (basal stems slightly blackened and leaves not wilted), 2 (basal stems blackened 1 to $2 \mathrm{~cm}$ and some leaves wilted), 3 (basal stems blackened $>2 \mathrm{~cm}$ and about half the leaves wilted), 4 (basal stems blackened and constricted and most leaves wilted), and 5 (the whole plant withered or dead). Each experiment was repeated twice, each time with 20 independent biological replicates.

\section{SIGS of RXLR effector genes in pepper and $N$. benthamiana leaves.}

$R X L R 1$-dsRNA, $R X L R 4$-dsRNA, and GFP-dsRNA were first in vitro-synthesized using a MEGAscript RNAi kit (Life Technologies) (Wang et al. 2016). Briefly, according to the manufacturer protocols, T7 promoter was introduced at both $5^{\prime}$ and $3^{\prime}$ ends of the DNA fragments by PCR, using primers listed in Supplementary Table $\mathrm{S} 1$. The specific DNA fragments of $R X L R I$ and $R X L R 4$, corresponding to the VIGS sequences described above, were used for in-vitro transcription. GFP-dsRNA, which derived from the GFP gene, served as a negative control. The synthesized dsRNAs were digested with ShortCut RNase III (New England Biolabs, Ipswich, MA, U.S.A.) to generate siRNAs and were subsequently purified using the mirVan miRNA isolation kit (Life Technologies). siRNAs (20 $\mu \mathrm{l}$, equivalent to $40 \mathrm{ng} / \mu \mathrm{l})$ were dropped onto the detached pepper or $N$. benthamiana leaves, followed one day later by $P$. capsici inoculation with mycelial plugs or $3 \mu \mathrm{l}$ of zoospores $\left(5 \times 10^{5}\right.$ per milliliter). The transcriptional expression of each RXLR gene was determined by qRT-PCR analysis at $1 \mathrm{dpi}$. We measured the disease lesions and relative $P$. capsici biomass at 2 to $3 \mathrm{dpi}$. Each experiment described above was repeated twice, each time with five independent biological replicates.

\section{ACKNOWLEDGMENTS}

We are grateful to T. Gbokie for his careful editing and helpful advice.

\section{LITERATURE CITED}

Barchenger, D. W., Lamour, K. H., and Bosland, P. W. 2018. Challenges and strategies for breeding resistance in Capsicum annuum to the multifarious pathogen, Phytophthora capsici. Front. Plant Sci. 9:628.

Basenko, E. Y., Pulman, J. A., Shanmugasundram, A., Harb, O. S., Crouch, K., Starns, D., Warrenfeltz, S., Aurrecoechea, C., Stoeckert, C. J., Jr., Kissinger, J. C., Roos, D. S., and Hertz-Fowler, C. 2018. FungiDB: An integrated bioinformatic resource for fungi and oomycetes. J. Fungi (Basel) 4:39.

Baulcombe, D. 2004. RNA silencing in plants. Nature 431:356-363.

Cai, Q., He, B., Kogel, K. H., and Jin, H. 2018. Cross-kingdom RNA trafficking and environmental RNAi-nature's blueprint for modern crop protection strategies. Curr. Opin. Microbiol. 46:58-64.

Cheng, W., Jiang, Y., Peng, J., Guo, J., Lin, M., Jin, C., Huang, J., Tang, W., Guan, D., and He, S. 2020. The transcriptional reprograming and functional identification of WRKY family members in pepper's response to Phytophthora capsici infection. BMC Plant Biol. 20:256.

Cheng, W., Lin, M., Qiu, M., Kong, L., Xu, Y., Li, Y., Wang, Y., Ye, W., Dong, S., He, S., and Wang, Y. 2019. Chitin synthase is involved in vegetative growth, asexual reproduction and pathogenesis of Phytophthora capsici and Phytophthora sojae. Environ. Microbiol. 21: 4537-4547

Cheng, W., Xiao, Z., Cai, H., Wang, C., Hu, Y., Xiao, Y., Zheng, Y. Shen, L., Yang, S., Liu, Z., Mou, S., Qiu, A., Guan, D., and He, S. 2017. A novel leucine-rich repeat protein, CaLRR51, acts as a positive regulator in the response of pepper to Ralstonia solanacearum infection. Mol. Plant Pathol. 18:1089-1100.

Dang, F. F., Wang, Y. N., Yu, L., Eulgem, T., Lai, Y., Liu, Z. Q., Wang, X., Qiu, A. L., Zhang, T. X., Lin, J., Chen, Y. S., Guan, D. Y., Cai, H. Y., Mou, S. L., and He, S. L. 2013. CaWRKY40, a WRKY protein of pepper, plays an important role in the regulation of tolerance to heat stress and resistance to Ralstonia solanacearum infection. Plant Cell Environ. 36:757-774.

Dubrovina, A. S., and Kiselev, K. V. 2019. Exogenous RNAs for gene regulation and plant resistance. Int. J. Mol. Sci. 20:2282.

Fan, G., Yang, Y., Li, T., Lu, W., Du, Y., Qiang, X., Wen, Q., and Shan, W. 2018. A Phytophthora capsici RXLR effector targets and inhibits a plant PPIase to suppress endoplasmic reticulum-mediated immunity. Mol. Plant 11:1067-1083.

Fernandez-Pozo, N., Rosli, H. G., Martin, G. B., and Mueller, L. A. 2015. The SGN VIGS tool: User-friendly software to design virusinduced gene silencing (VIGS) constructs for functional genomics. Mol. Plant 8:486-488.

Hannon, G. J. 2002. RNA interference. Nature 418:244-251.

He, Q., McLellan, H., Boevink, P. C., and Birch, P. R. J. 2020. All roads lead to susceptibility: The many modes of action of fungal and oomycete intracellular effectors. Plant Commun. 1:100050.

Jackson, K. L., Yin, J., and Ji, P. 2012. Sensitivity of Phytophthora capsici on vegetable crops in Georgia to mandipropamid, dimethomorph, and cyazofamid. Plant Dis. 96:1337-1342.

Jahan, S. N., Åsman, A. K. M., Corcoran, P., Fogelqvist, J., Vetukuri, R. R., and Dixelius, C. 2015. Plant-mediated gene silencing restricts growth of the potato late blight pathogen Phytophthora infestans. J. Exp. Bot. 66:2785-2794.

Jiang, R. H. Y., and Tyler, B. M. 2012. Mechanisms and evolution of virulence in oomycetes. Annu. Rev. Phytopathol. 50:295-318.

Kamoun, S., Furzer, O., Jones, J. D. G., Judelson, H. S., Ali, G. S., Dalio, R. J. D., Roy, S. G., Schena, L., Zambounis, A., Panabières, F., Cahill, D., Ruocco, M., Figueiredo, A., Chen, X. R., Hulvey, J., Stam, R., Lamour, K., Gijzen, M., Tyler, B. M., Grünwald, N. J., Mukhtar, M. S., Tomé, D. F., Tör, M., Van Den Ackerveken, G., McDowell, J., Daayf, F., Fry, W. E., Lindqvist-Kreuze, H., Meijer, H. J., Petre, B. Ristaino, J., Yoshida, K., Birch, P. R., and Govers, F. 2015. The top 10 oomycete pathogens in molecular plant pathology. Mol. Plant Pathol. 16:413-434.

Kenski, D. M., Butora, G., Willingham, A. T., Cooper, A. J., Fu, W., Qi, N., Soriano, F., Davies, I. W., and Flanagan, W. M. 2012. siRNA-optimized modifications for enhanced in vivo activity. Mol. Ther. Nucleic Acids 1:e5.

Ketting, R. F. 2011. The many faces of RNAi. Dev. Cell 20:148-161.

Koch, A., Biedenkopf, D., Furch, A., Weber, L., Rossbach, O., Abdellatef, E., Linicus, L., Johannsmeier, J., Jelonek, L., Goesmann, A., Cardoza, V., McMillan, J., Mentzel, T., and Kogel, K. H. 2016. An RNAi-based control of Fusarium graminearum infections through spraying of long dsRNAs involves a plant passage and is controlled by the fungal silencing machinery. PLoS Pathog. 12:e1005901.

Koch, A., and Kogel, K. H. 2014. New wind in the sails: Improving the agronomic value of crop plants through RNAi-mediated gene silencing. Plant Biotechnol. J. 12:821-831

Lamour, K. H., Mudge, J., Gobena, D., Hurtado-Gonzales, O. P., Schmutz, J., Kuo, A., Miller, N. A., Rice, B. J., Raffaele, S., Cano, L. M., Bharti, A. K., Donahoo, R. S., Finley, S., Huitema, E., Hulvey, J., Platt, D., Salamov, A., Savidor, A., Sharma, R., Stam, R., Storey, D., Thines, M., Win, J., Haas, B. J., Dinwiddie, D. L., Jenkins, J., Knight, J. R., Affourtit, J. P., Han, C. S., Chertkov, O., Lindquist, E. A., Detter, C., Grigoriev, I. V., Kamoun, S., and Kingsmore, S. F. 2012b. Genome sequencing and mapping reveal loss of heterozygosity as a mechanism for rapid adaptation in the vegetable pathogen Phytophthora capsici. Mol. Plant-Microbe Interact 25:1350-1360.

Lamour, K. H., Stam, R., Jupe, J., and Huitema, E. 2012a. The oomycete broad-host-range pathogen Phytophthora capsici. Mol. Plant Pathol. 13:329-337.

Li, Q., Ai, G., Shen, D., Zou, F., Wang, J., Bai, T., Chen, Y., Li, S., Zhang, M., Jing, M., and Dou, D. 2019b. A Phytophthora capsici 
effector targets ACD11 binding partners that regulate ROS-mediated defense response in Arabidopsis. Mol. Plant 12:565-581.

Li, Q., Chen, Y., Wang, J., Zou, F., Jia, Y., Shen, D., Zhang, Q., Jing, M., Dou, D., and Zhang, M. 2019a. A Phytophthora capsici virulence effector associates with NPR1 and suppresses plant immune responses. Phytopathol. Res. 1:6.

Li, Q., Wang, J., Bai, T., Zhang, M., Jia, Y., Shen, D., Zhang, M., and Dou, D. 2020. A Phytophthora capsici effector suppresses plant immunity via interaction with EDS1. Mol. Plant Pathol. 21:502511.

Li, T., Wang, Q., Feng, R., Li, L., Ding, L., Fan, G., Li, W., Du, Y., Zhang, M., Huang, G., Schäfer, P., Meng, Y., Tyler, B. M., and Shan, W. 2019c. Negative regulators of plant immunity derived from cinnamyl alcohol dehydrogenases are targeted by multiple Phytophthora Avr3a-like effectors. New Phytol.

Livak, K. J., and Schmittgen, T. D. 2001. Analysis of relative gene expression data using real-time quantitative PCR and the $2^{-\Delta \Delta C(T)}$ method. Methods 25:402-408.

Mitter, N., Worrall, E. A., Robinson, K. E., Li, P., Jain, R. G., Taochy, C., Fletcher, S. J., Carroll, B. J., Lu, G. Q., and Xu, Z. P. 2017. Clay nanosheets for topical delivery of RNAi for sustained protection against plant viruses. Nat. Plants 3:16207.

Mo, H., Kim, S., Wai, K. P. P., Siddique, M. I., Yoo, H., and Kim, B. 2014. New sources of resistance to Phytophthora capsici in Capsicum spp. Hortic. Environ. Biotechnol. 55:50-55.

Nowara, D., Gay, A., Lacomme, C., Shaw, J., Ridout, C., Douchkov, D., Hensel, G., Kumlehn, J., and Schweizer, P. 2010. HIGS: Host-induced gene silencing in the obligate biotrophic fungal pathogen Blumeria graminis. Plant Cell 22:3130-3141.

Nunes, C. C., and Dean, R. A. 2012. Host-induced gene silencing: A tool for understanding fungal host interaction and for developing novel disease control strategies. Mol. Plant Pathol. 13:519-529.

Qiao, L., Lan, C., Capriotti, L., Ah-Fong, A., Nino Sanchez, J., Hamby, R., Heller, J., Zhao, H., Glass, N. L., Judelson, H. S., Mezzetti, B., Niu, D., and Jin, H. 2021. Spray-induced gene silencing for disease control is dependent on the efficiency of pathogen RNA uptake. Plant Biotechnol. J. 19:1756-1768.

Reyes-Tena, A., Huguet-Tapia, J. C., Lamour, K. H., Goss, E. M., Rodríguez-Alvarado, G., Vázquez-Marrufo, G., Santillán-Mendoza, R., and Fernández-Pavía, S. P. 2019. Genome sequence data of six isolates of Phytophthora capsici from Mexico. Mol. Plant-Microbe Interact 32:1267-1269.
Ryu, C. M., Anand, A., Kang, L., and Mysore, K. S. 2004. Agrodrench: A novel and effective agroinoculation method for virus-induced gene silencing in roots and diverse Solanaceous species. Plant J. 40:322-331.

Sanju, S., Siddappa, S., Thakur, A., Shukla, P. K., Srivastava, N., Pattanayak, D., Sharma, S., and Singh, B. P. 2015. Host-mediated gene silencing of a single effector gene from the potato pathogen Phytophthora infestans imparts partial resistance to late blight disease. Funct. Integr. Genomics 15:697-706.

Senthil-Kumar, M., and Mysore, K. S. 2014. Tobacco rattle virus-based virus-induced gene silencing in Nicotiana benthamiana. Nat. Protoc. 9:1549-1562

Tyler, B. M., Tripathy, S., Zhang, X., Dehal, P., Jiang, R. H., Aerts, A., Arredondo, F. D., Baxter, L., Bensasson, D., Beynon, J. L., Chapman, J., Damasceno, C. M., Dorrance, A. E., Dou, D., Dickerman, A. W., Dubchak, I. L., Garbelotto, M., Gijzen, M., Gordon, S. G., Govers, F., Grunwald, N. J., Huang, W., Ivors, K. L., Jones, R. W., Kamoun, S., Krampis, K., Lamour, K. H., Lee, M. K., McDonald, W. H., Medina, M., Meijer, H. J., Nordberg, E. K., Maclean, D. J., Ospina-Giraldo, M. D., Morris, P. F., Phuntumart, V., Putnam, N. H., Rash, S., Rose, J. K., Sakihama, Y., Salamov, A. A., Savidor, A., Scheuring, C. F., Smith, B. M., Sobral, B. W., Terry, A., Torto-Alalibo, T. A., Win, J., Xu, Z., Zhang, H., Grigoriev, I. V., Rokhsar, D. S., and Boore, J. L. 2006. Phytophthora genome sequences uncover evolutionary origins and mechanisms of pathogenesis. Science 313:1261-1266.

Vega-Arreguín, J. C., Jalloh, A., Bos, J. I., and Moffett, P. 2014. Recognition of an Avr3a homologue plays a major role in mediating nonhost resistance to Phytophthora capsici in Nicotiana species. Mol. PlantMicrobe Interact 27:770-780.

Wang, K., Peng, Y., Chen, J., Peng, Y., Wang, X., Shen, Z., and Han, Z 2020. Comparison of efficacy of RNAi mediated by various nanoparticles in the rice striped stem borer (Chilo suppressalis). Pestic. Biochem. Physiol. 165:104467.

Wang, M., and Dean, R. A. 2020. Movement of small RNAs in and between plants and fungi. Mol. Plant Pathol. 21:589-601.

Wang, M., and Jin, H. 2017. Spray-induced gene silencing: A powerful innovative strategy for crop protection. Trends Microbiol. 25:4-6.

Wang, M., Weiberg, A., Lin, F. M., Thomma, B. P., Huang, H. D., and Jin, H. 2016. Bidirectional cross-kingdom RNAi and fungal uptake of external RNAs confer plant protection. Nat. Plants 2:16151.

Waterhouse, P. M., Wang, M. B., and Lough, T. 2001. Gene silencing as an adaptive defence against viruses. Nature 411:834-842. 